\title{
Disk evaporation in a planetary nebula ${ }^{\star}$
}

\author{
K. Gesicki ${ }^{1}$, A. A. Zijlstra ${ }^{2}$, C. Szyszka ${ }^{3,2}$, M. Hajduk ${ }^{1,4}$, E. Lagadec ${ }^{3}$, and L. Guzman Ramirez ${ }^{2}$ \\ 1 Centrum Astronomii UMK, ul.Gagarina 11, 87-100 Torun, Poland \\ e-mail: Krzysztof.Gesicki@astri.uni.torun.pl \\ 2 Jodrell Bank Centre for Astrophysics, School of Physics \& Astronomy, University of Manchester, Oxford Road, \\ Manchester M13 9PL, UK \\ e-mail: a.zijlstra@manchester.ac.uk \\ 3 European Southern Observatory, Karl Schwarzschildstrasse 2, Garching 85748, Germany \\ ${ }^{4}$ Nicolaus Copernicus Astronomical Center, ul. Rabiańska 8, 87-100 Torun, Poland
}

Received 24 October 2009 / Accepted 20 January 2010

\section{ABSTRACT}

\begin{abstract}
Aims. Binary interactions are believed to be important contributors to the structures seen in planetary nebulae (PN), and the sole cause of the newly discovered compact dust disks. The evolution of these disks is not clear, nor are the binary parameters required for their creation.

Methods. Using HST imaging and VLT spectroscopy, both long-slit and integral field, we study the Galactic bulge planetary nebula M 2-29 for which a 3-year eclipse event of the central star has been attributed to a dust disk.

Results. The central PN cavity of M 2-29 is being filled with a decreasingly, slow wind. An inner high density core is detected, of radius smaller than $250 \mathrm{AU}$, interpreted as a rotating gas/dust disk with a bipolar disk wind. The evaporating disk is argued to be the source of the slow wind. The central star is the source of a very fast wind $\left(\sim 10^{3} \mathrm{~km} \mathrm{~s}^{-1}\right)$. An outer, partial ring is seen in the equatorial plane, expanding at $12 \mathrm{~km} \mathrm{~s}^{-1}$. The azimuthal asymmetry is attributed to mass-loss modulation by an eccentric binary. A crucial point in disk evolution is represented by M2-29 where ionization causes the gas to be lost, leaving a low-mass dust disk behind.
\end{abstract}

Key words. ISM: planetary nebulae: individual: M 2-29 (PN G 004.0-03.0) - stars: AGB and post-AGB ISM: planetary nebulae: general

\section{Introduction}

Disks are a recent addition to the range of structures seen in planetary nebulae. The outer nebulae show a large variety of morphological characteristics, including tori, bipolar flows, and ellipsoidal shapes (e.g., Ramos-Larios et al. 2008), with typical sizes of $10^{4}-10^{5} \mathrm{AU}$. But embedded in these nebulae a central dust disk has sometimes been discovered with sizes of order $10^{2} \mathrm{AU}$, which can be resolved with the VLT interferometer (e.g., Chesneau et al. 2007). The disks appear well aligned with the outer nebulae but are of too low mass to have directly affected their formation. They are more likely to be a byproduct of the original shaping process.

Other evidence exists for the presence of material very close to some central stars, including unresolved emission-line cores (Rodriguez et al. 2001) and eclipses from dust disks (Hajduk et al. 2008). The disks are believed to require binary systems for their formation but the details of the required system parameters are unclear. An interesting review of binary systems and their shaping of PNe was published by de Marco (2009).

Here we present a study of a planetary nebula with such an unresolved emission-line core, known to harbour a dust disk: M 2-29 (PN G 004.0-03.0). Deep echelle spectra and integralfield spectra, together with HST imaging, allow us to disentangle the various components of this complex nebula, and to determine the velocity fields. We find the central core to be the source of

^ Based on observations collected at the European Organisation for Astronomical Research in the Southern Hemisphere, Chile (proposals 075.D-0104, 077.D-0652, 081.D-0130) and HST (program 9356). an ongoing, but decreasing wind, which we attribute to a disk wind. Although direct evidence of the binary companion remains elusive, the outer structure suggests an interaction with a star on a non-circular orbit. From comparing with other objects with dust disks, we find evidence that these disks strongly decrease in mass during the planetary nebula evolution. However, they remain sufficiently massive for residual disks to survive long into the white dwarf phase.

\section{Observations}

\subsection{The HST images}

SNAPshot HST/WFPC2 images of M 2-29 were obtained in 2003, in three different filters: F656N, F547M, and F502N. The pixel scale of the camera is 0.0455 arcsec. The $\mathrm{H} \alpha$ image (Hajduk et al. 2008) is show in Fig. 1. The main nebular components are the central source, a wing-like structure, and the extended, fainter nebula.

\subsection{VLT/UVES long-slit spectra}

A 600-s VLT/UVES (Dekker et al. 2000) echelle spectrum was obtained in 2005, using a slit 0.5 arcsec wide and 11 arcsec long. The slit was placed through the centre of the nebula, aligned with the jet-like structure. The pipeline calibration was applied, including wavelength calibration and merging of the separate orders of the spectrum. The observations cover a spectral region approximately from 3300 to $6600 \AA$ A. The long-slit spectra 


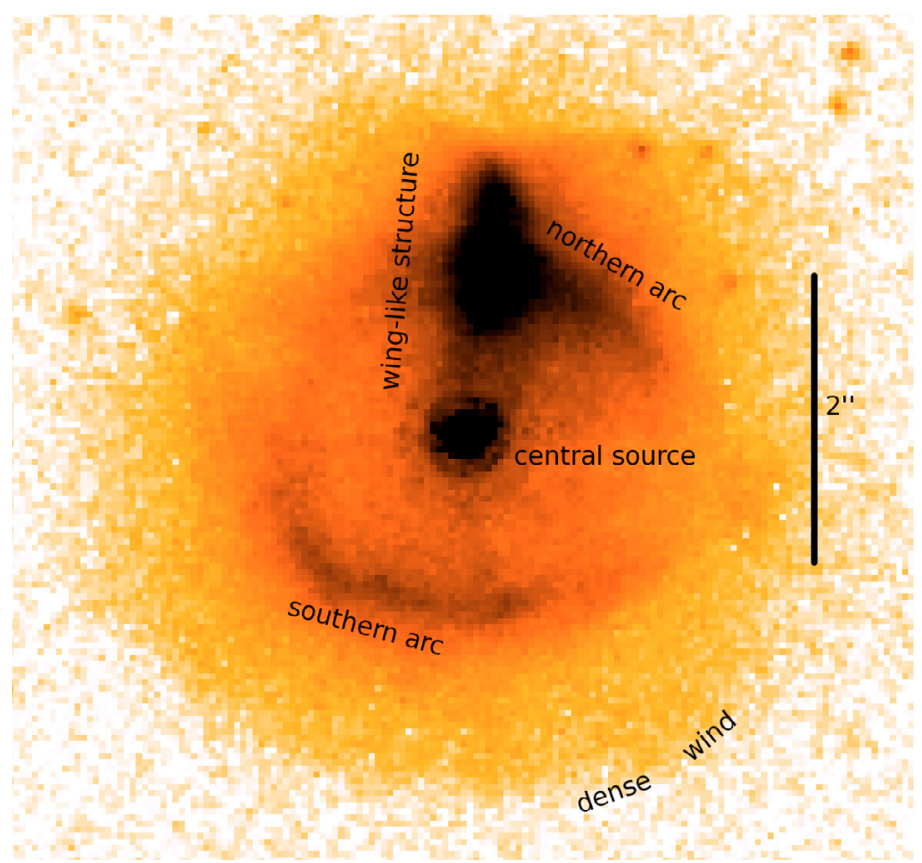

Fig. 1. H $\alpha$ HST image of M 2-29 obtained in 2003. Left shows a linear intensity scale, right shows a logarithmic intensity scale to bring out the fainter emission. North is on top, east to the left. The length of the bar is 2 arcsec. Taken from Hajduk et al. (2008).

of M2-29 are spatially resolved, although they are at much poorer resolution than HST images and the seeing during the observations was nearly 1.5 arcsec.

\subsection{VLT/ARGUS-IFU spatially resolved spectroscopy}

We also retrieved publicly available data from the ARGUS integral field unit (IFU), a mode of the FLAMES instrument at the VLT (Pasquini et al. 2002). These observations of M2-29 were performed in visitor mode as part of program 077.D-0652 (PI. D. Schönberner). Two spectral ranges are available, HR8 and HR14B, that cover the ranges 4911-5158 $\AA$ and 6383-6626 with resolution $R \sim 32000$ and $R \sim 46000$, respectively. Because we were targeting for small-scale structure, we selected only those frames of seeing superior to 1 arcsec; this constraint yielded one frame in HR14 (700 s) and six frames in HR8 $(6 \times$ $110 \mathrm{~s})$. The large-area $\left(12 \times 7 \operatorname{arcsec}^{2}\right)$ IFU was used, where each single resolving element (spaxel) covers $0.52 \times 0.52 \operatorname{arcsec}^{2}$.

The data were analyzed with the GIRAFFE base line data reduction software (girBLDRS a.k.a. Geneva pipeline). Correction of bias-level flat-fielding, and wavelength calibration were prepared in a standard way. Spectra were extracted with the sumextraction method, because the default optimum extraction is not well suited to an emission-like object. The airmass was lower than 1.06 for all frames alleviating the need to correct for differential atmospheric refraction. The data were not flux calibrated.

\subsection{VLT/VISIR images}

Detected at $25 \mu \mathrm{m}$ by IRAS, M 2-29 is a known dust emitter, its $12-\mu \mathrm{m}$ detection having been rejected from the faint-source catalogue, perhaps because of confusion with a nearby star. $N$-band spectroscopy presented by Casassus et al. (2001) is indicative of a possible broad silicate emission band at $8-12 \mu \mathrm{m}$. We retrieved and reduced archival Spitzer IRS data, which confirms that silicate emission is present, and shows a weak PAH feature at $11.2 \mu \mathrm{m}$ (Fig. 2).
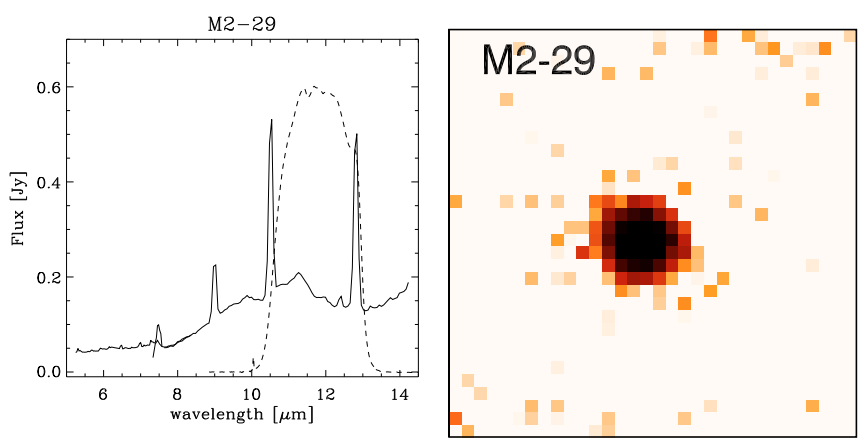

Fig. 2. Left: Spitzer low-resolution spectrum, showing a broad silicate band with superimposed emission lines and a weak $11.2 \mu \mathrm{m}$ PAH band. Right: the VISIR image showing a strong point source. The image is $3.8 \times 3.8$ arcsec in size. The transmission curve of the filter used is shown on the spectrum.

To study the dust location we obtained mid-infrared images of M 2-29 using VISIR on the VLT (Lagage et al. 2004). We used a 0.075 arcsec per pixel scale and the VISIR SiC filter $\left(\lambda_{\mathrm{c}}=11.85 \mu \mathrm{m}, \Delta \lambda=2.34 \mu \mathrm{m}\right)$. The observations were carried out in visitor mode with high quality weather conditions, leading to diffraction-limited images during the whole run. The observations were performed using the standard chopping and nodding technique to reduce the background emission in the midinfrared. The data reduction was performed using self-developed IDL routines described by Lagadec et al. (2008). Images were corrected for bad pixels and then coadded to produce a single flat-field-corrected image, comprising the average of the chop and nod differences.

The resulting image at $11.85 \mu \mathrm{m}$ shows an unresolved source (Fig. 2), indicating that the emitting source is smaller than $\sim 0.3$ arcsec. Thus, the emitting hot dust only originates in one compact component (the core), and not from the extended nebula or wing-like feature. 
K. Gesicki et al.: Disk evaporation in a planetary nebula

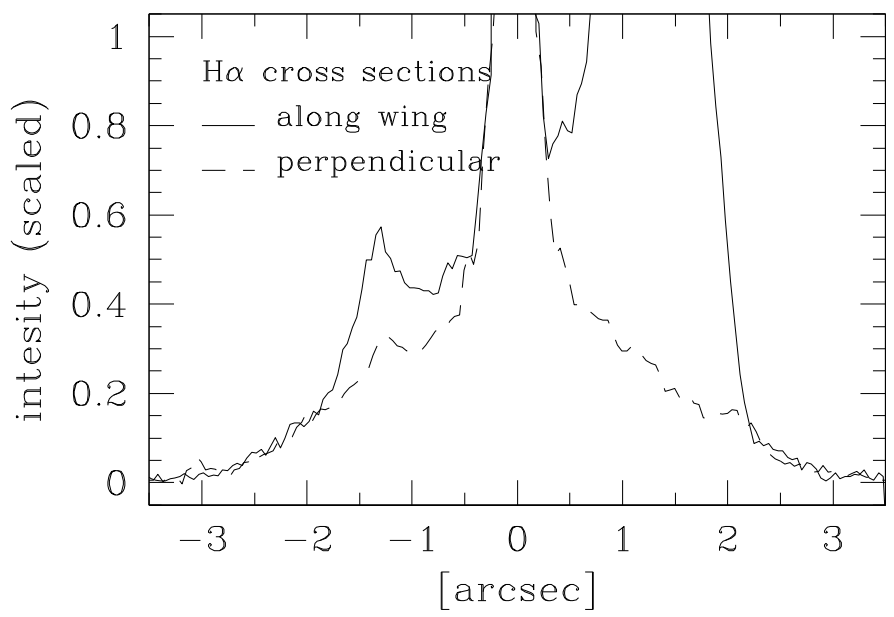

Fig. 3. The cross-sections through the $\mathrm{H} \alpha$ HST image of M 2-29 taken along the wing-like structure and perpendicularly to it. The intensity scale is the same for both profiles.

\section{Morphology from HST images}

Shown in Fig. 1, the HST H $\alpha$ image (Hajduk et al. 2008) illustrates the complexity of the nebula, including the main ring, the halo, the wing-like structure, and the unresolved central object. The main ring is composed of two arcs, which may not quite connect. The gaps between them are nearly perpendicular to the wing-like structure. There is a slight indication of elongation or brightening in the EW direction, beyond the gaps (most clearly visible in the left panel, with a linear scale). The overall impression is that of a barrel-shaped nebula, with the beginnings of a bipolar flow, a relatively common shape among planetary nebulae.

The HST H $\alpha$ and [O III] $5007 \AA$ images are almost identical and have the same extent. This means that M 2-29 should be fully ionized (density bounded). In the discussion below, we refer to the $\mathrm{H} \alpha$ image.

\subsection{The outer halo and dense wind}

To inspect the outer regions, we overplot in Fig. 3 two scaled cross-sections: one extracted along the wing-like structure and the other perpendicularly to it. The outermost nebular regions decrease in brightness in the same way in all directions, which is indicative of spherical symmetry. There is no clear evidence of ISM interaction (Wareing et al. 2007).

The emission profile interestingly extends smoothly and symmetrically inside the arcs, out to the central object. This is shown in (Fig. 3), where the small bump on the left hand side corresponds to the arcs. The arcs and jet appear to be superimposed on a largely spherical component whose brightness smoothly decreases outward. We refer to this component as the "dense wind". The spherical symmetry of this component points to a stellar origin. Its brightness in the $\mathrm{H} \alpha$ image proves that it is not hydrogen deficient.

The emission profile of the dense wind, including its halo, corresponds roughly to the surface brightness of a sphere filled by matter with a $r^{-0.5}$ density distribution. This is unusual. The most widely adopted AGB wind density is $r^{-2}$, as discussed by Villaver et al. (2002). The $r^{-0.5}$ density implies a mass-loss rate that decreases as $\dot{M} \propto t^{-1.5}$. The surface brightness with an expansion velocity of $10 \mathrm{~km} \mathrm{~s}^{-1}$ infers a current mass loss rate of $10^{-8} M_{\odot} \mathrm{yr}^{-1}$.

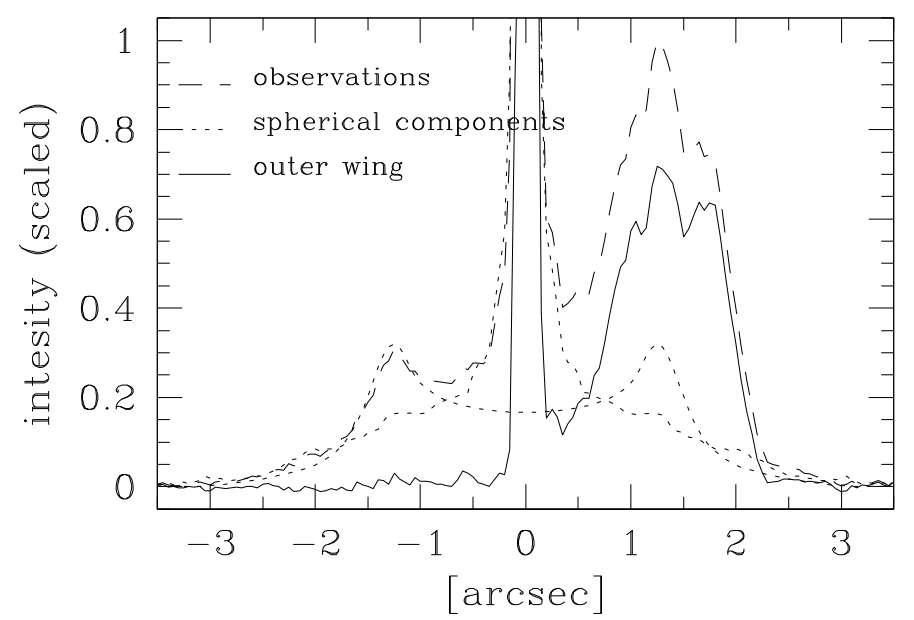

Fig. 4. The cross-sections through the H $\alpha$ HST image of M2-29 taken along the wing-like structure. The dashed line shows the observed intensity profile, the dotted lines indicate both spherical components (the dense wind and the arcs), and the continuous line presents the difference between the observed cross-section and the two spherical components.

\subsection{The central nebula}

Using the HST images, Hajduk et al. (2008) showed that the unresolved source at the centre contains an ionized nebula that outshines the central star in terms of emission lines.

A number of artificial PSFs were created using the TinyTim v.6.3 (Krist \& Hook 2004), and fitted to this central nebula. Series of sub-pixel images for different shifts relative to the nebular image were obtained. The PSFs were convolved with Gaussian kernels of different FWHMs, and resampled to the resolution of the Planetary Camera. After resampling, the charge diffusion kernel was applied. The PSF was then subtracted from the M 2-29 image. Minimum residuals were obtained for PSFs convolved with a Gaussian of $F W H M \sim 0.8$ pixel. A similar result was obtained for a background star. The broadening of the PSF with respect to the theoretical one may be caused by an imperfect focus. By comparing with the field star, we constrain the deconvolved $F W H M$ of the nebula to be $<0.6$ pixel. At the distance of $8 \mathrm{kpc}$ this corresponds to a diameter of $<250 \mathrm{AU}$ $\left(3 \times 10^{15} \mathrm{~cm}\right)$.

\subsection{The wing-like feature}

To obtain the radial emission profile of the wing-like feature from the HST image, the other nebular components should be subtracted.

The profile of the dense wind was obtained from the dashed line in Fig. 3, by taking the average of this cross-section with its mirror reflection. The profiles of the arcs are more difficult to obtain, as they are not circularly symmetric and cannot be taken from the perpendicular profiles. We instead used a photoionization model (see Sect. 5), approximated by fitting a spherical shell to the two arcs, and subtracting the profile calculated from this model. The unresolved core was not subtracted. During decomposition, weighting factors were applied. The result is shown in Fig. 4.

To the left (south) of the central object, a flat profile was obtained. The wing-like structure shows up only to the north (right) of the core. 


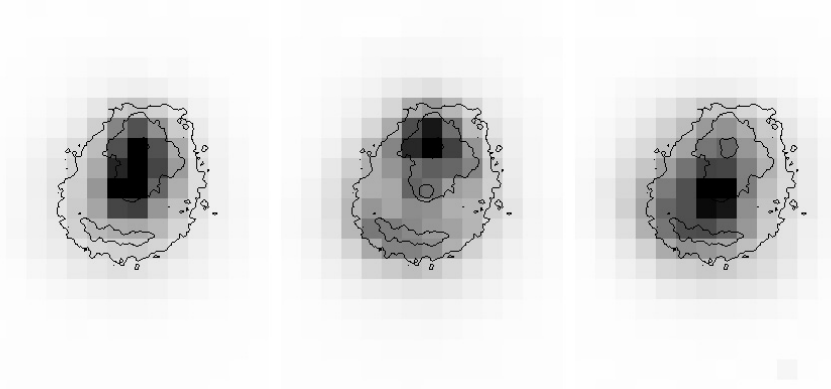

(a) $[\mathrm{O}$ III $] 5007 \AA$ at $-12 \mathrm{~km} \mathrm{~s}^{-1}$

(b) $[\mathrm{O}$ III] $5007 \AA$ at system velocity

(c) $[\mathrm{O} \mathrm{III]}] 5007 \AA$ at $+12 \mathrm{~km} \mathrm{~s}^{-1}$

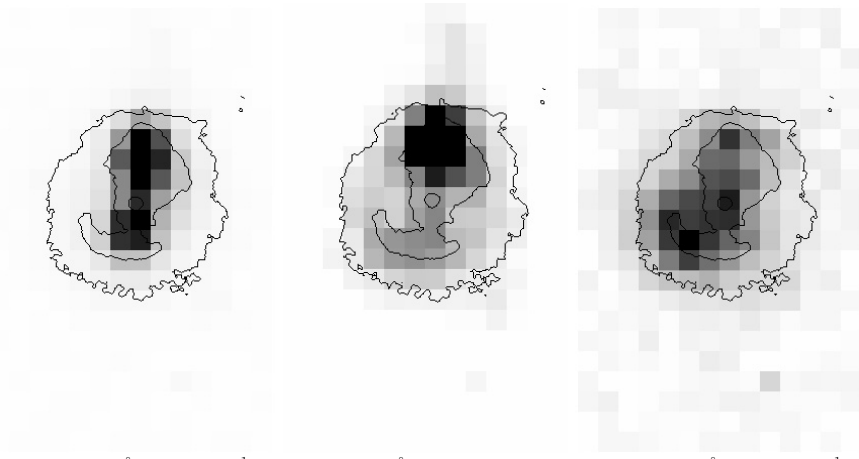

(e) $\left[\mathrm{N}\right.$ II] $6583 \AA$ at $-12 \mathrm{~km} \mathrm{~s}^{-1}$

(f) $\left[\mathrm{N}_{\mathrm{II}}\right] 6583 \AA$ at system velocity

(g) $\left[\mathrm{N}\right.$ II] $6583 \AA$ at $+12 \mathrm{~km} \mathrm{~s}^{-1}$
.

(d) $[\mathrm{O}$ III $] 5007 \AA ̊$ long-slit spectrum
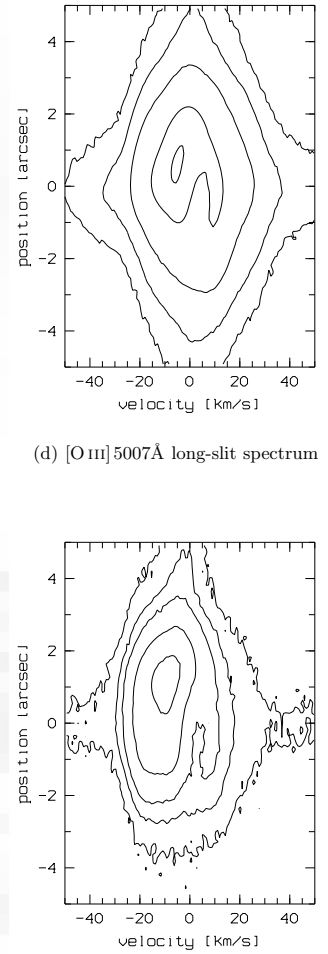

(h) $\left[\mathrm{N}_{\mathrm{II}}\right] 6583 \AA$ long-slit spectrum
Fig. 5. The ARGUS-IFU and UVES observations of M2-29 in spectral lines of [O III] $5007 \AA$ (upper row) and [N II] $6583 \AA$ (lower row). The presented slices (left) are extracted from ARGUS data cubes at wavelengths corresponding to velocity shifts from the systemic velocity of $-12 \mathrm{~km} \mathrm{~s}^{-1}, 0$, and $+12 \mathrm{~km} \mathrm{~s}^{-1}$ (from left to right respectively). The fluxes are shown in grey in linear scale. The black colour corresponds to the relative intensity of 0.9 except of panels e) and f), where cuts were applied at levels 0.5 and 0.3 , respectively. In both lines, the image at $-12 \mathrm{~km} \mathrm{~s}^{-1}$ (panels a) and e) is the brightest. For [O III], the central image b) is of similar brightness, while $\mathbf{c}$ ) is two times weaker. For [N II], panels f) and $\mathbf{g}$ ) are fainter by a factor of 3 and 30 with respect to panel e). The contours are from the HST images, overplotted to the same spatial scale; for the [O III] slices, the [O III] image is shown, while for the [N II] slices the $\mathrm{H} \alpha$ image is shown. The right-most panels show the UVES long-slit spectra, where the slit was positioned along the wing-like structure, i.e., nearly N-S direction. The contours are 0.004 , $0.02,0.1,0.5$, and 0.95 for $[\mathrm{O} \mathrm{III]}]$ in panel d) and $0.007,0.03,0.08,0.3$, and 0.8 for $[\mathrm{N} \mathrm{II}]$ in panel h).
No clear residual is seen at the cross-over point of the winglike feature and the arcs. A superposition on the sky, rather than a physical connection, seems to be indicated.

\subsection{The arcs}

The successful decomposition of the image cross-section into the dense wind and the photo-ionization model, shown in Fig. 4, indicates that the southern arc can be interpreted as a fragment of a photoionized sphere showing emissivity enhancement at the edge. The same can be said about the northern arc. A very common interpretation of this structure is that the arcs project in the form of a thin barrel, open at the two poles. The dense wind fills both the inside and the outside of the barrel. This appears to suggest that the density enhancement of the barrel formed out of the dense wind.

\section{The spectra}

\subsection{Velocity maps}

In Fig. 5, we present spectroscopic observations in emission lines of [O III] $5007 \AA$ and [N II] $6583 \AA$. These are strong forbidden lines, which probe the inner and outer nebular regions, respectively. For both lines, we extracted from the data cubes three representative slices corresponding to the systemic radial velocity (panels $b$ and $\mathrm{f})^{1}$ and those shifted by $12 \mathrm{~km} \mathrm{~s}^{-1}$ below (panels a and e) and above (panels $\mathrm{c}$ and g) this value. The greyscale represents the relative intensities normalized for each slice separately. The square pixels are of spatial size $0.52 \times 0.52 \mathrm{arcsec}^{2}$. The contours delineate the HST images: [O III] for the upper row,

\footnotetext{
1 The velocity is corrected for the radial velocity of the object of $-112.2 \mathrm{~km} \mathrm{~s}^{-1}$ (Durand et al. 1998) and barycentric velocity for the moment of observations of $-10.4 \mathrm{~km} \mathrm{~s}^{-1}$ for UVES and $-22 \mathrm{~km} \mathrm{~s}^{-1}$ for ARGUS.
}

$\mathrm{H} \alpha$ for the bottom row. The right-most frames show the long-slit UVES spectra, where the slit was positioned along the wing-like structure, i.e., $5^{\circ}$ away from the $\mathrm{N}-\mathrm{S}$ direction.

At the central star position, the [O III] velocity maps exhibit strong emission at both the positive and negative velocities (panels a and c) but a minimum at the systemic velocity (panel b). This emission corresponds to the unresolved central component and can be interpreted in terms of a bipolar outflow. The winglike structure can be traced in panels (a) and (b) with velocity decreasing outwards; this can also be seen in the long-slit spectra (panel d). The southern arc is most clearly identifiable in the zero velocity panel (b).

More details can be derived from [N II] images. The winglike structure at $-12 \mathrm{~km} \mathrm{~s}^{-1}$ (panel e) is clearly more extended than in [O III] at the same velocity (panel a), and is seen at both sides of the central component. The systemic velocity image (panel f) shows the wing-like structure extending two arcseconds in the northern direction, far beyond what is seen in the $\mathrm{H} \alpha$ image. The long-slit UVES spectra (panel h) also show this significant extension. The southern arc can also be discerned at the systemic velocity, and a very weak component is seen in panel (f) extending for a couple of pixels in the S-W direction. Panel (g) shows that a component at the eastern end of the southern arc is moving away from us, at a little more than $+12 \mathrm{~km} \mathrm{~s}^{-1}$.

In Fig. 6, we present more examples of the 2-D (positionvelocity) contours presentation of bright emission lines in the UVES spectrum. All plots are shown to the same scale, but in the blue spectral region the slit was shorter so the spatial extent of these lines is smaller. Many more emission lines are identifiable in the spectrum, but the three presented here are those that complement most closely those in Fig. 5, which shows the variety of line profiles.

The emission of the wing-like feature is visible from the centre until about 2 arcsec up. It is very pronounced in the [O III] $5007 \AA$, [N II] (Fig. 5), and He I lines, very weak in 


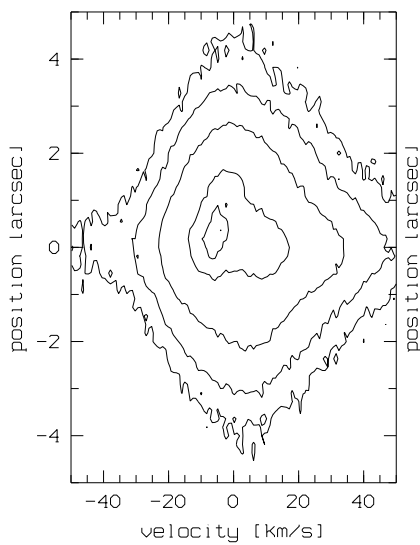

(a) HeI $5876 \AA$

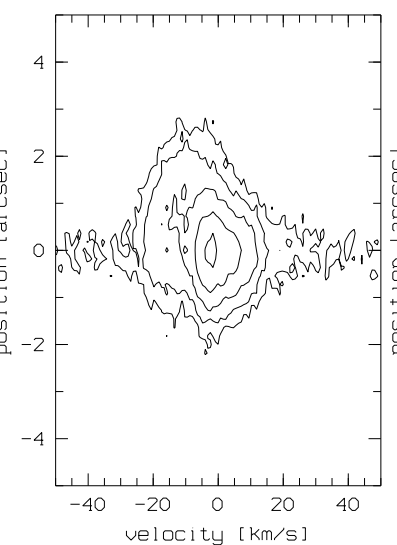

(b) O I $6300 \AA$

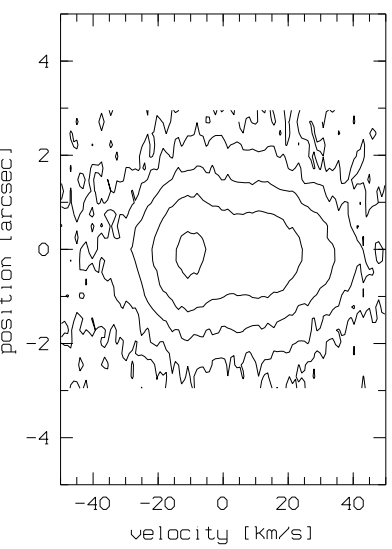

(c) $[\mathrm{O}$ III $] 4363 \AA$
Fig. 6. The VLT/UVES long-slit spectrum of He I $5876 \AA$ (left), [O I] $6300 \AA$ (middle), and [O III] $4363 \AA$ (right). The velocity scale is centred on the systemic velocity. The wing-like structure extends up from the centre. The contours are at levels of $0.02,0.07,0.2,0.7$, and 0.9 in panel a), 0.06, 0.1, 0.2, 0.5, and 0.9 in panel b) and $0.01,0.03,0.1,0.3$, and 0.8 in panel c).

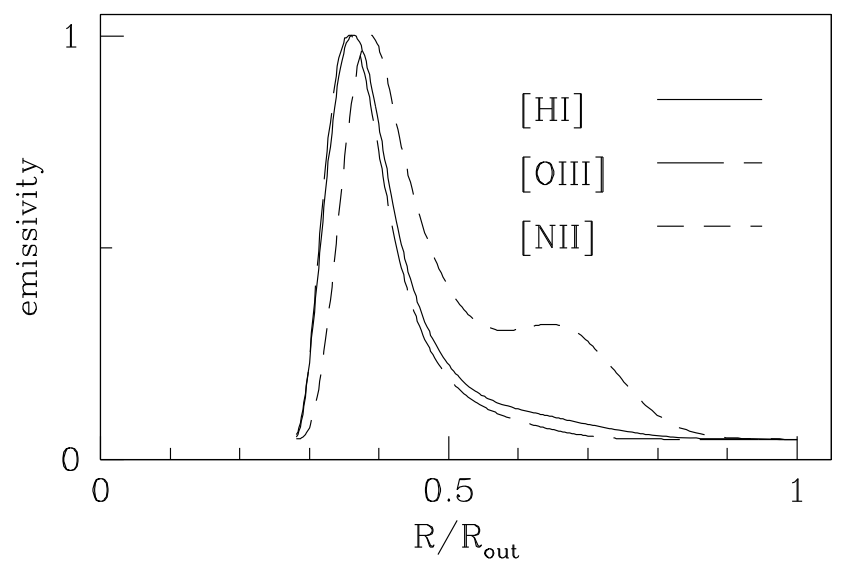

Fig. 7. Model intensity distribution for H I, O III, and N II. The overall density follows $\mathrm{H}$ to high accuracy.

the $[\mathrm{OI}]$ line, and completely absent in the highly excited [O III] $4363 \AA$ line.

The velocities are relatively low (around $10 \mathrm{~km} \mathrm{~s}^{-1}$ ) compared to the typical expansion velocities of planetary nebulae.

\subsection{Photoionization modelling of the outer nebula}

To model the spectra and line profiles, we applied the Torun photoionization codes (Gesicki \& Zijlstra 2003; Gesicki et al. 2006). The star is assumed to be a black body with a luminosity and effective temperature. The nebula is approximated as a spherical shell defined by the radial density distribution and radial velocity field. The chemical composition $([\mathrm{O} / \mathrm{H}]=7.44)$ and observed line intensities were adopted from Exter et al. (2004). We also measured the line intensities from the low resolution SAAO spectrum (Hajduk et al. 2008). Table 1 presents the observed data with our best-fit model. The model emissivities are shown in Fig. 7.

The one-dimensional model by necessity assumes spherical symmetry. It solves for the observed line intensities and the radial surface brightness distribution. For the latter, we extracted the image cross-sections taken at 45 degrees left and right to the wing-like structure, i.e., avoiding the wing while including the arcs. The two cross-sections are quite similar. The model assumes a central cavity: neither the central unresolved nebula, nor the inner part of the dense wind (i.e., inside the arcs) is included.

The satisfactory solution is summarized in Table 2. Although the comparison of the $\mathrm{H} \alpha$ and [O III] HST images indicates a
Table 1. Dereddened M2-29 emission-line flux ratios, relative to $I(\mathrm{H} \beta)=100.0$.

\begin{tabular}{llrrrr}
\hline \hline $\begin{array}{l}\text { Wavel. } \\
{[\AA]}\end{array}$ & Ident. & \multicolumn{3}{c}{ Observed flux } & Model \\
{$[\AA]$} & & SAAO & \multicolumn{1}{c}{$(1)$} & \multicolumn{1}{c}{$(2)$} & \\
\hline 3726 & {$[\mathrm{O} \mathrm{II}]$} & 43.6 & 42.1 & 34 & 15.8 \\
3869 & {$[\mathrm{Ne}$ III $]$} & 72.2 & 49.8 & 58 & 19.7 \\
4363 & {$[\mathrm{O} \mathrm{III}]$} & 27.0 & 14.0 & - & 1.9 \\
4959 & {$[\mathrm{O} \mathrm{III}]$} & 150.2 & 146.0 & - & 46.8 \\
5007 & {$[\mathrm{O} \mathrm{III}]$} & 449.4 & 424.0 & 452 & 134.9 \\
6300 & {$[\mathrm{O} \mathrm{I}]$} & 1.6 & 1.1 & - & 0.4 \\
6548 & {$[\mathrm{~N} \mathrm{II}]$} & 7.0 & 6.9 & - & 10.2 \\
6584 & {$[\mathrm{~N} \mathrm{II}]$} & 17.8 & 20.8 & 17 & 29.9 \\
6716 & {$[\mathrm{~S} \mathrm{II}]$} & 1.2 & 1.9 & - & 0.7 \\
6731 & {$[\mathrm{~S} \mathrm{II}]$} & 1.8 & 2.4 & 2 & 0.8 \\
7136 & {$[\mathrm{Ar}$ III $]$} & 9.0 & 12.5 & - & 8.3 \\
\hline
\end{tabular}

Notes. Observed data come from our SAAO spectrum (dereddened with $c_{\beta}=1.18$ (Hajduk et al. 2008)) and from literature (dereddened by the authors).

References. (1) Exter et al. (2004); (2) Howard et al. (1997).

density bounded PN, the line intensities (integrated over the entire nebula) are significantly more closely fitted when the PN is ionization bounded. We solved this problem by adopting the density distribution extending far beyond the arcs, obtaining the ionization front at about 4 arcsec radius. This ensures that the model differs slightly from that in Hajduk et al. (2008). The strong [O III] $5007 \AA$ line is not closely fitted. We could only fit this line by increasing the oxygen abundance by a factor of 2 , which remains metal-poor however we did not attempt to improve the adopted abundance determination.

The model has a constant expansion velocity with distance from the star with simultaneous broadening by turbulence, as indicated by the similar widths of lines formed at different radii. This is at variance with the usual positive velocity gradients seen in planetary nebulae. The model turbulence probably reflects the superposition of different nebular components. The massaveraged expansion velocity derived for M 2-29 is $12 \mathrm{~km} \mathrm{~s}^{-1}$ (compared to $14 \mathrm{~km} \mathrm{~s}^{-1}$ obtained from [O III] only, Gesicki \& Zijlstra 2000). Assuming a distance of $8 \mathrm{kpc}$, this velocity results in a kinematic age of about $5000 \mathrm{yr}$.

The ionized mass of the nebula is rather high, in view of the conclusion of Torres-Peimbert et al. (1997) about old age (and therefore low initial mass) of the object. The age may have been overestimated, or mass transfer could instead have been 
Table 2. M 2-29 photoionization and kinematic model parameters.

\begin{tabular}{ll}
\hline \hline Parameter & Value \\
\hline Distance & $8 \mathrm{kpc}$ \\
$T_{\text {eff }}$ & $70000 \mathrm{~K}$ \\
Luminosity & $1200 \mathrm{~L}_{\odot}$ \\
Ionized mass & $0.38 \mathrm{M}_{\odot}$ \\
$\log \mathrm{He} / \mathrm{H}+12$ & 10.99 \\
$\log \mathrm{N} / \mathrm{H}+12$ & 7.14 \\
$\log \mathrm{O} / \mathrm{H}+12$ & $7.44^{*}$ \\
$\log \mathrm{Ne} / \mathrm{H}+12$ & 6.84 \\
$\log \mathrm{S} / \mathrm{H}+12$ & 5.64 \\
Nebula expansion velocity & $12 \mathrm{~km} \mathrm{~s}^{-1}$ \\
Turbulent velocity component & $7 \mathrm{~km} \mathrm{~s}^{-1}$ \\
Kinematic age & $5000 \mathrm{yr}$ \\
\hline
\end{tabular}

Notes. ${ }^{(*)}$ This adopted value might be too low (see the text).

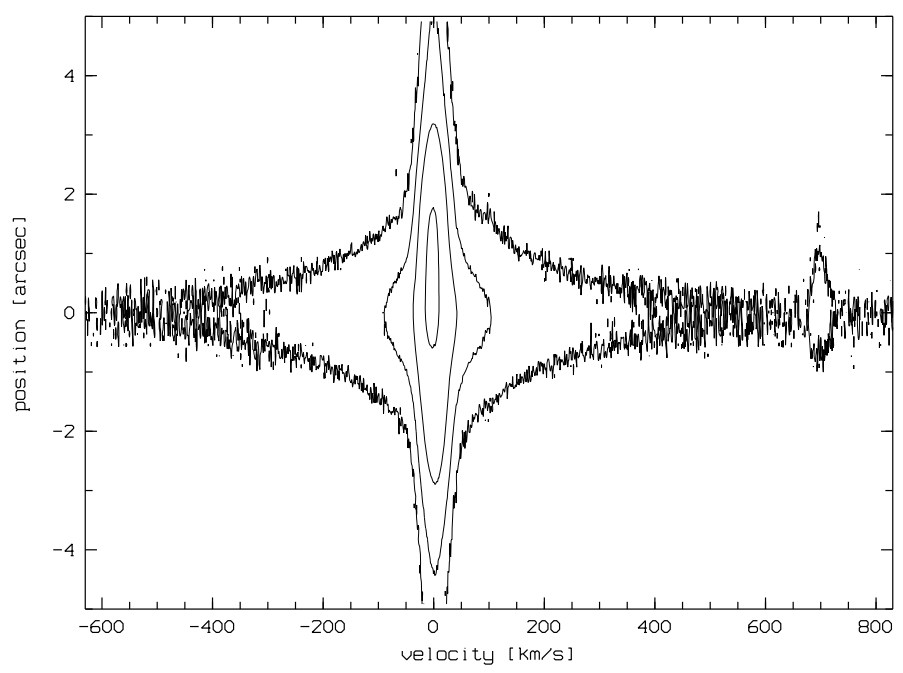

Fig. 8. The $P-V$ plot of the $\mathrm{H} \alpha$ line observed with UVES, the longslit being aligned with the wing-like structure. At the right edge, the permitted line of C II $6578 \AA$ can be seen. Contours are equally spaced in logarithmic intensity.

proposed. However, caution should be taken when deriving a mass from a spherical model for a non-spherical nebula.

\subsection{Extended wings of $\mathrm{H} \alpha$}

The $\mathrm{H} \alpha$ emission line exhibits very wide wings, seen at the location of the central unresolved source only. In the UVES position-velocity diagram (Fig. 8), the vertical structure shows the emission from the wing-like structure, and the horizontal extent shows the high velocity wings, located at the same position as the stellar continuum.

Averaging the slit spectra over the central 1.5 arcsec, the emission wings can be traced over $1000 \mathrm{~km} \mathrm{~s}^{-1}$ (Fig. 9). The ARGUS-IFU data cube confirms that at wavelengths beyond $\pm 4 \AA\left( \pm 200 \mathrm{~km} \mathrm{~s}^{-1}\right)$ from the line centre the emission originates solely in the unresolved source.

Extended wings can also be seen in [O III] $5007 \AA$ as shown in Fig. 5d, and in $\mathrm{H} \beta$ (not shown here). For weaker lines, it is unclear whether the wings are present or absent (see e.g., N II $6583 \AA$ line in Fig. 5h). However, the O III $5007 \AA$ line exhibits wings one order of magnitude narrower than $\mathrm{H} \alpha$.

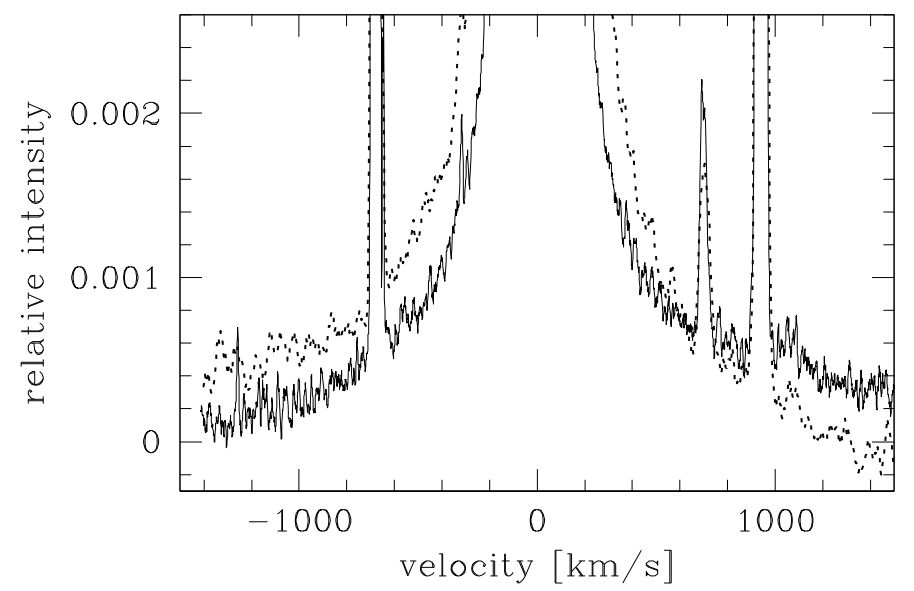

Fig. 9. The $\mathrm{H} \alpha$ line extracted from the central pixels of UVES (solid line) and ARGUS-IFU (dotted line). The emission is normalized to the line peak. Note that ARGUS has a lower spectral resolution.

This should be considered when interpreting this emission component.

Evidence against electron scattering is that the large broadening is seen only in some lines. A high velocity component in the central object is indicated.

\subsection{The permitted emission lines}

The UVES spectrum exhibits a number of permitted emission lines. The recombination lines of C II are present at 4267.26 and $6578.05 \AA$ (e.g., Fig. 8). The recombination lines of O III at $4638.85,4641.81,4649.14$, and $4650.84 \AA$ can also be identified. These lines are useful in abundance analysis (e.g., Garcia-Rojas et al. 2005).

For the clearly detected C II $6578 \AA$ line, an ARGUS-IFU image was obtained by integrating over its line width. This line originates in an extended region as can be seen in Fig. 10 where it is compared with He I $5016 \AA$ line. The situation for the other permitted lines is not so clear because they are weaker and noisier. The well-known group around $4650 \AA$ falls at an echelleorder merging where distortions may occur; it is also not covered by the ARGUS-IFU spectral range. The UVES data suggests that it may also form in an extended region but this requires confirmation.

We also identified a number of permitted N III lines (4097.33, 4103.43, 4634.14, 4640.64, 4641.85 $\AA$ ). They are often interpreted in terms of the Bowen mechanism (see e.g., Selvelli et al. 2007), where He II Ly $\alpha$ overlaps with an O III line and the resulting O III cascade itself has a frequency that coincides with an N III resonance line. The O III Bowen lines below $4000 \AA$ fall within a very noisy area of our VLT spectrum and cannot be identified. The very weak He II line argues against a Bowen mechanism operating in the nebula of M2-29. Mihalas (1971) proposed that dielectronic recombination could explain the N III line complex near $4650 \AA$. The N III lines are only detected from the central nebula.

\subsection{Interstellar absorption and distance}

The UVES spectra of M2-29 show interstellar absorption lines of $\mathrm{NaI}$ and $\mathrm{Ca}$ II, as shown in Fig. 11.

The equivalent widths (EW) of the $\mathrm{CaIIH}$ and $\mathrm{K}$ lines (i.e., adding together all Doppler components) exhibit an 


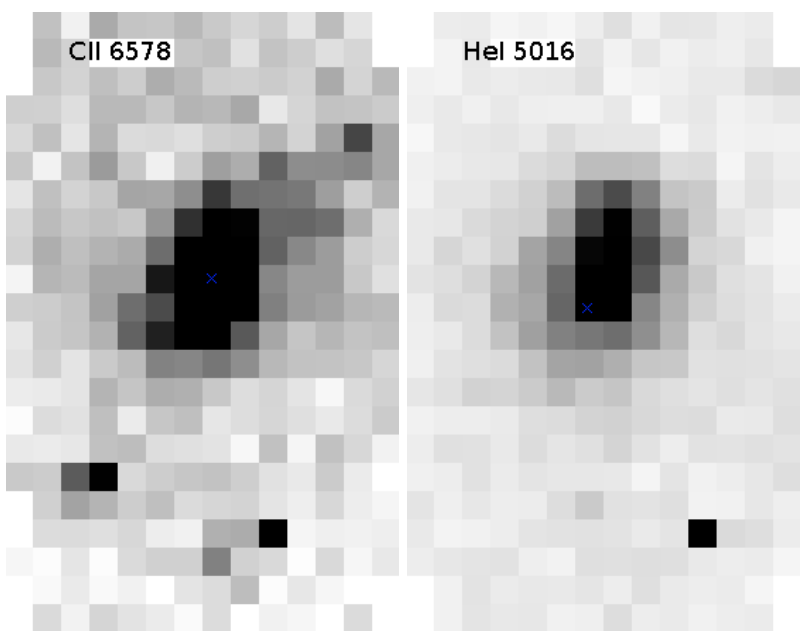

Fig. 10. The ARGUS-IFU collapsed images of the CII $6578 \AA$ and He I $5016 \AA$ lines. North is on top, east to the left, the size of a single pixel is 0.52 arcsec.

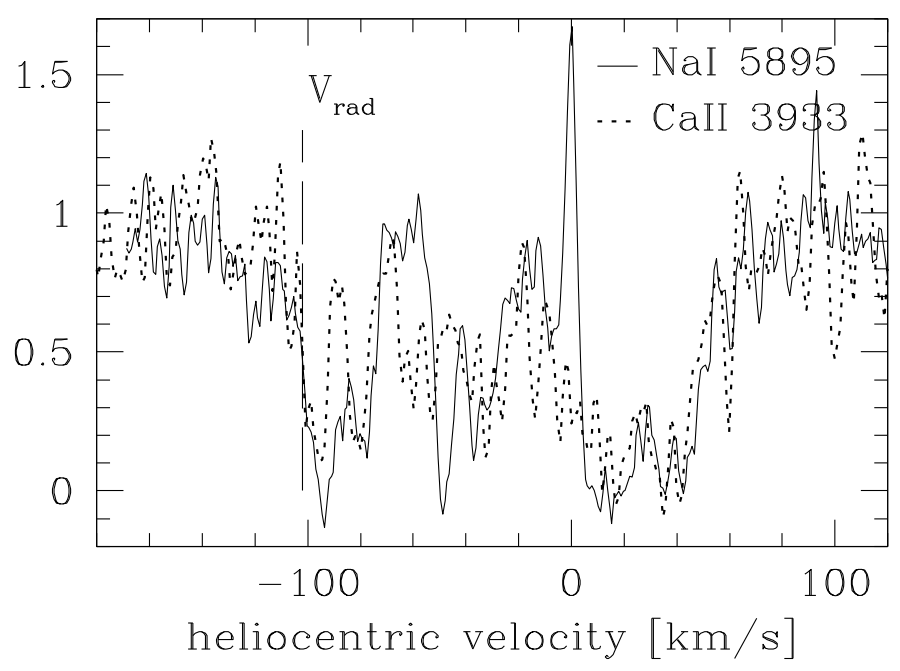

Fig. 11. The interstellar absorption line profiles of ions $\mathrm{Na}$ I and $\mathrm{Ca}$ II $\mathrm{K}$ in the spectrum of M2-29. The profiles are shown in the heliocentric reference frame.

approximately linear relation with distance (Megier et al. 2005), as derived from OB stars with Hipparcos parallaxes. For M 2-29, the measured EW of the full $\mathrm{Ca}$ II $\mathrm{K}$ absorbing complex is about $1500 \mathrm{~m} \AA$ (the $H$-band being confused with $\mathrm{H} \epsilon$ ). This results in a distance of 4 or $6 \mathrm{kpc}$ when applying the relations of Megier et al. (2005) or Galazutdinov (2005), respectively. It is unclear whether the linear relation can be extrapolated to the inner regions of the Galaxy but the values appear to infer a Galactic bulge location.

The interstellar absorption-line profiles contain components for different interstellar clouds. The three main components are at (heliocentric) velocities of $0-50 \mathrm{~km} \mathrm{~s}^{-1}$, at -60 to $-20 \mathrm{~km} \mathrm{~s}^{-1}$, and at -100 to $-80 \mathrm{~km} \mathrm{~s}^{-1}$. The absorption at positive velocities can be associated with the molecular ring around the inner Galaxy, while the intermediate negative velocity component is consistent with the $3 \mathrm{kpc}$ expanding ring detected in $\mathrm{CO}$ (Dame et al. 2001). However, the Galactic CO maps show no counterpart to the most negative velocity component in M2-29. This component can be explained by neither Galactic rotation, nor bulge rotation ${ }^{2}$.

HIPASS spectra in this region show an $\mathrm{HI}$ emission feature corresponding to this negative velocity component. It may be related to a nearby catalogued high velocity cloud, HVC 002.906.2-088 (Putman et al. 2002), of the same velocity, because the emission at this velocity seems to extend over quite some distance. The distance to this cloud or complex is not known, but as M 2-29 is clearly located behind it, we suggest that it is situated relatively close to the Galactic centre. Small Ca II and $\mathrm{Na}$ I absorption clouds with similar high velocities appear to be common in the Galactic halo (Ben Bekhti et al. 2008), having a variety of origins.

It is remarkable that the non-rotational interstellar absorption component near $-100 \mathrm{~km} \mathrm{~s}^{-1}$ is very close to the systemic radial velocity of M 2-29. As a PN, or related object, M 2-29 is expected to be old and evolved, and any association with the interstellar component is deemed accidental.

\section{Identifying the components of the nebula}

The nebula of M2-29 appears to contain four distinct components: the inner unresolved nebula, the dense wind extending outwards, the arcs, and the wing-like structure. The arcs appear to be an enhancement within the dense wind rather than an independent structure. Here we attempt to understand the form of the other two structures.

\subsection{An outer partial ring: the wing-like structure}

The "wing-like" feature is a low-velocity structure. Based on its observed location and lack of interaction with the arc, we locate it outside of the main nebula. We identify it as a partial, expanding ring, surrounding the main nebula, whose plane is almost coincident with the line of sight. The ring has an inner radius of about 2 arcsec.

The velocity structure along this ring is depicted in Figs. 5 and 6. In the [O III] line, only the top (north) of the ring is seen, but the $[\mathrm{N}$ II] line shows the more complete structure. In the southern part, the [N II] is displaced to the left (see Fig. $5 \mathrm{~g}$ ). The displacement suggests that the plane of the ring is seen at an inclination of approximately 20 degrees with respect to the line of sight. Because the displacement is seen in the rear part of the ring, it is not unlikely that the structure is warped.

The line splitting indicates that it is expanding: an expansion velocity (with a minor correction for the inclination) of about $12 \pm 2 \mathrm{~km} \mathrm{~s}^{-1}$ is indicated.

The UVES slit was aligned with the brightest part of this structure, although because of the inclination the southern extent fell outside the slit. This is clear from the ARGUS image. The position-velocity diagrams for the UVES data therefore have incomplete coverage. Nevertheless, the brightness distribution of the ring is strongly asymmetric, while the velocity profiles appear to be symmetric. The peak [O III] emission occurs at a velocity of $-10 \mathrm{~km} \mathrm{~s}^{-1}$ and is offset by about 1 arcsec from the star. This corresponds to a location on the ring about 30 degrees from the line of sight to the centre. The bright [O III] emission is superimposed on an arc of roughly 90 degrees, on the forward side of the ring. Some faint emission originates in the backside of the ring, as is clearly evident towards the southwest at positive velocities, in Fig. 5c.

\footnotetext{
2 The mean rotation of the Galactic bulge at the longitude of M 2-29 is around $+50 \mathrm{~km} \mathrm{~s}^{-1}$ (Minniti \& Zoccali 2008).
} 
The bright [N II] emission covers a much wider arc, extending for some 120 degrees. At a much fainter level, the ring may be almost closed. The [N II] emission is also spatially far more extended than [O III], containing a fainter outer region of radius at least 4 arcsec. This is seen in Fig. $5 f$ as the northern extension. This region only contributes at near-systemic velocity, suggesting that the expansion velocity decreases sharply from 2 to 4 arcsec radius. The radial extension is not seen towards the south, showing that at its outermost radius the ring is not closed and perhaps limited to some 180 degrees.

The ring is also seen in $\mathrm{He}$ I, C II, and [O I] at the location of the brightest [O III] and [N II] emission. Thus, there is sufficient column density in this direction to cause ionization stratification and an ionization boundary, as also indicated by the photoionization model. The lack of [O III] beyond $2 \operatorname{arcsec}$ is consistent with the photoionization model of Fig. 7.

Finally, this ring system is located close to the equatorial plane of the main nebular barrel defined by the northern and southern arc (see Sect. 3). A slight misalignment is explained by the inclination.

Thus, we arrive at a model of an expanding equatorial, partial ring, 2-4 arcsec in radius, with a strong azimuthal density gradient, a radial density gradient, and an expansion velocity of $12 \pm 2 \mathrm{~km} \mathrm{~s}^{-1}$ at 2 arcsec, which decreases with increasing radius.

\subsection{The unresolved disk}

For the central source, we detected the emission lines of $\mathrm{H} \alpha$, [O III] 4363 and $5007 \AA$, He II, the Bowen N III lines, and [O I]. Other lines are present but are less clearly detected because of the spatial and velocity overlap with the extended emission. The very high velocity wings and the [O III] $4363 \AA$ line are seen only in this core. The size of the core is $<0.03 \mathrm{arcsec}$, or $<250 \mathrm{AU}$ at the distance of the Galactic bulge.

The [O III] 4363/5007 line ratio is much higher in the core than in the extended nebula. ${ }^{3}$ This is indicative of high density and temperature, in agreement with Torres-Peimbert et al. (1997) who first detected a high density and high electron temperature component close to the star. We ran some test models to estimate the parameters of the inner nebula, using the central star parameters derived above. In these models, the ratio 4363/5007 [O III] lines can be reproduced by assuming that $T_{\mathrm{e}} \approx 1.4 \times 10^{4} \mathrm{~K}$ and $n_{\mathrm{e}} \approx 6 \times 10^{5} \mathrm{~cm}^{-3}$. These values are a little lower than the estimates of Torres-Peimbert et al. (1997). The mass of the compact nebula at these densities should be $10^{-4} M_{\odot}$ to reproduce the observed $\mathrm{H} \beta$ flux. This value is not very accurate, as the core is unlikely to be of constant density and spherical symmetry, but it provides a believable order of magnitude. Other lines often used as electron density indicators, e.g., lower ionized/excited [O II] or [S II], originate predominantly in the winglike structure. This ensures that an extraction of the central component becomes far more unreliable, in no case as clear as the [O III] $4363 \AA$ line.

All lines exhibit velocity broadening of $\sim 10 \mathrm{~km} \mathrm{~s}^{-1}$. For this velocity, the kinematical age of the core is younger than 100 years, requiring a continuing mass-loss rate of $\sim 10^{-6} M_{\odot} \mathrm{yr}^{-1}$. The lower limit to the mass-loss rate is 1 to 2 orders of magnitude lower than that required for the extended nebula (assuming a kinematic age of $5000 \mathrm{yr}$ ), and the surface brightness distribution of the extended wind indeed implies a

\footnotetext{
3 In the photoionization model of the outer nebula, the [O III] $4363 \AA$ line is underestimated by an order of magnitude, which is another argument for it not belonging to the outer main ring.
}

linearly decreasing mass-loss rate with time. It remains unclear, however, why the mass-losing star is not seen. Such mass-loss rates and velocities are found in red giants, where the wind from the hot central star is very much faster. Neither the spectrum nor the photometry shows evidence of a contribution from any star other than the PN central star. The 2MASS photometry excludes the presence of a red giant more luminous than $100 L_{\odot}$. Although a mass-losing star has clearly been present in the recent past, it does not appear to be present at the moment.

The alternative to ongoing stellar mass-loss is a stable, rotating structure, which may be supported by a measured decrease in the line widths with decreasing excitation, from $36 \mathrm{~km} \mathrm{~s}^{-1}$ $F W H M$ for [O III] line to close to a few $\mathrm{km} \mathrm{s}^{-1}$ or smaller for the $[\mathrm{OI}]$ line, these values being consistent with rotation velocities an outward decrease. Hajduk et al. (2008) derive an orbital period for the wide binary of $\sim 17 \mathrm{yr}$, implying an orbital velocity of approximately $10-15 \mathrm{~km} \mathrm{~s}^{-1}$. They also demonstrate that a circumbinary dust disk in this system can explain the longduration eclipse. A rotating, circumbinary disk would exhibit an innermost velocity a little less than the binary orbital velocity, at some $10 \mathrm{AU}$, in agreement with [O III]. The [O I] velocity corresponds to a value inferred by the model to occur at radii of a few hundred AU.

The extreme $\mathrm{H} \alpha$ wings are indicative of a fast outflow, which in a stable disk will produce a bipolar flow pattern. The dense wind shows the presence of outflow: we interpret this as a disk wind, caused by the evaporation of the photoionized gas. The limit of stability of the gas disk is set when the thermal velocities exceed the rotational velocity, beyond about $100 \mathrm{AU}$, only neutral gas or dust being stable. Evaporating gas can be accelerated by the fast $\mathrm{H} \alpha$ wind, as a mass-loaded wind (Borkowski et al. 1995). The [O III] core emission exhibits a double peak, and this may indicate that it forms partly in such a bipolar wind.

It is highly probably that the inner nebula is, therefore, an opaque disk seen edge-on (Hajduk et al. 2008) with ionized bipolar outflows. The infrared dust emission and neutral [OI] line originate in the outer static boundary. The dense wind originates in the evaporating disk. At larger distances, this evaporating-disk wind is expected to be spherically symmetric (Alexander 2008). The disk can be located in the same plane as the outer partial ring, at an inclination of 20 degrees from the line of sight, the bipolar flow being directed towards the opening between the two arcs.

\section{Discussion}

\subsection{The nebula}

The one-side structure of the wing in M 2-29 is unusual but not unique. It is duplicated in both He 2-428, and A 79 (Rodriguez et al. 2001), and possibly also in the symbiotic star V417 Cen (Van Winckel et al. 1994). In all four cases, the structure is almost identical, allowing for the difference in viewing angle. However, no further object with a similar morphology could be identified in the IAC morphological catalog (Manchado et al. 1996), confirming the rarity of this structure.

Outer asymmetries can be formed by interactions with the interstellar medium, which are common occurrences (Wareing et al. 2007). In M 2-29, there is indeed a possible association with interstellar gas at a similar velocity, and a possible ISM sweep-up should be explored. However, the good alignment of the outer ring with the equatorial plane of the nebula, the presence of expansion, and the existence of a few very similar 
objects, all suggest that the partial ring was part of the planetary nebula ejection.

The arcs appear to be related to a more (if not entirely) spherically symmetric component in the nebula, located within the outer ring. This suggests a scenario where the mass ejection was initially highly asymmetric, but became less so over time. The arcs could trace a temporary mass-loss enhancement, or they could be caused by the pressure enhancement from the onset of ionization. The compact core is evidence that a fraction of the ejected mass was retained close to the star.

The ejection of the partial ring may be related to an interaction with a companion in an elliptical orbit. This interaction is discussed by Soker \& Rappaport (2001), their scenario 4. If the orbit is such that interaction occurs at periastron only, amplifying or initiating the mass loss at this point, the mass loss will occur preferentially in the direction of the velocity vector of the mass-losing star at periastron, and not at the systemic velocity. As the stellar radius increases, the binary orbit may circularize or the stellar mass loss may become self-sustaining: the mass loss will now occur everywhere along the orbit, centred on the systemic velocity.

The interaction between these two winds offset in velocity (Soker \& Rappaport 2001) provides a natural explanation of why an identical structure is seen in several objects, and seems the most plausible explanation for the outer wing, and for the asymmetry occurring only during early mass loss. There is some evidence that V417 Cen, which exhibits a similar morphology, has an elliptical orbit (Van Winckel et al. 1994).

\subsection{Classifying the star}

The PN M 2-29 is one of only three known to experience dust eclipses, the others being NGC 2346 and CPD-56 8032 (Hajduk et al. 2008; Cohen et al. 2002; De Marco et al. 2002), and one of two PNe with a binary period of order 1 month (NGC 2346 has a period of 16 days, Hajduk et al. 2008; de Marco 2009).

It is also one of a few showing a dense, unresolved nebular core inside an extended PN. Other cases include EGB 6, a very faint and nearly circular PN around a binary composed of a very hot white dwarf and a visual point-like companion with dense emission-line nebula (Bond et al. 1993). Rodriguez et al. (2001) finds a similar core in He 2-428. A mixed class including some young PNe (Lamers et al. 1998), B[e] stars, also appear to have compact cores and $\mathrm{H} \alpha$ line wings similar to M 2-29 (Zickgraf 2003). However, M 2-29 lacks the low-ionization iron lines found in $\mathrm{B}[\mathrm{e}]$ stars. A very late thermal pulse leads to the ejection of a new core (van Hoof et al. 2007), but this core is hydrogen-poor and differs from what is seen in M 2-29.

A relation to dusty symbiotic stars was proposed (Hajduk et al. 2008; Miszalski et al. 2009), based on the orbital period of M 2-29. Symbiotic systems contain a mass-losing giant, and a hot white dwarf that irradiates the wind: the ionized gas exhibits dense cores (e.g., M 2-9, OH 231.8+04.2). Although possibly related, M 2-29 does not, however, appear to be a symbiotic star. It shows neither the Raman lines at $6830 \& 7082 \AA$, nor any indications of a cool (giant or yellow) companion in the spectrum. Although there seems to be two companions in M2-29, (Hajduk et al. 2008), neither appears to be the required mass-losing giant.

However, M 2-29 will likely be a symbiotic star at some time. If the companion is a white dwarf, the object would have been a symbiotic star or symbiotic Mira in the recent past, while losing mass. If the companion is main sequence, it will become a symbiotic once the companion ascends the giant branches (e.g., de Marco 2009).

\subsection{Evolution of the disk}

Compact dust disks are common around post-AGB stars (de Ruyter et al. 2006), and being identified increasingly often around the central stars of planetary nebulae: examples are the ant nebula (Chesneau et al. 2007), the Helix nebula ( $\mathrm{Su}$ et al. 2007), CPD -56 8032 (Cohen et al. 2002), M 2-48 (Phillips \& Ramos-Larios 2008), and NGC 2346 (Costero et al. 1986).

The origin and evolution of these disks remains unclear. They are interpreted as circumbinary disks that formed during the mass-loss phase, or as debris disks either left over from the main sequence phase or built recently from tidally disrupted smaller orbiting bodies as discussed in e.g., Su et al. (2007) or Brinkworth et al. (2009). The plethora of different classes of objects with these disks (B[e] stars, symbiotic stars, RV Tau stars) indicates that they form relatively easily. Binary companions appear required in all cases, but the orbital periods range from 200 days (post-AGB binaries with disks) to $100 \mathrm{yr}$ (such as M 2-9).

Interestingly, similar dust disks have not been discovered around the post-common-envelope binaries seen in about 15-20\% of PNe (Miszalski et al. 2009), which have periods of hours to days. Although models (Sandquist et al. 1998; Nordhaus \& Blackman 2006) indicate that the common envelope is not necessarily completely ejected and some gas remains around the system, there is no evidence that this residual leads to a dust disk. The known dust disks are seen around longer-period systems that avoided a common envelope phase.

It is argued that M 2-29 corresponds to the phase where the gas disk is lost and the dust disk remains. Rotation velocities far exceed thermal velocities, while the gas is molecular or neutral. The dust settles towards the mid plane at this time (Dominik et al. 2003). However, once ionization starts, the thermal velocities are comparable to the rotation velocities and a disk wind initiates. In M 2-29, the disk wind began $\sim 10^{3} \mathrm{yr}$ ago, but has been decreasing over time, likely reflecting the decreasing gas reservoir. At the current time, some $10^{-4} M_{\odot}$ of gas remains. The current disk-wind mass-loss rate of $10^{-8} M_{\odot} \mathrm{yr}^{-1}$ infers a disk decay time similar to the age of the nebula.

The future evolution is of interest to the disks seen around more evolved objects. The Spitzer spectrum with the strong silicate emission and PAH emission indicates that the dust is relatively hot. The dust will cool sharply once the star enters the white dwarf cooling track where it rapidly fades by a factor of 100. The central star of the helix is such a cooling track star. The cooler dust will emit mainly at longer wavelengths: the dust disk of the helix is detected mainly at $24 \mu \mathrm{m}$ and $70 \mu \mathrm{m}$ (Su et al. 2007). These cool disks may have gone largely unnoticed.

Table 3 lists the ages and disk dust masses for four planetary nebulae where these have been measured. The ages are defined from the end of the AGB, i.e., the ejection of the main nebula. The dust masses are taken from the given references, where we assumed a gas-to-dust ratio of 100 for M2-29. The age of $\mathrm{CPD}-56^{\circ} 8032$ has probably been underestimated because the object is known for longer than this (Gill 1900).

The table indicates that the disk masses decrease sharply as the star ages. Although this result should be interpreted with care because of the small sample and the possibly disparate origins of the objects, it appears possible that much of the dust mass is lost during the PN phase. Dust can be carried away in the wind from the evaporating gas disk, and unshielded dust may 
Table 3. Ages and dust masses of the disks in planetary nebulae.

\begin{tabular}{lrrl}
\hline \hline Nebula & $\begin{array}{r}\text { Nebular Age } \\
{[\mathrm{yr}]}\end{array}$ & $\begin{array}{r}\text { Dust Mass } \\
{\left[M_{\odot}\right]}\end{array}$ & Reference \\
\hline CPD-56 8032 & $10^{2}$ & $3 \times 10^{-4}$ & $(2),(3)$ \\
Ant nebula (Mz 3) & $10^{3}$ & $1 \times 10^{-5}$ & $(1),(4)$ \\
M 2-29 & $5 \times 10^{3}$ & $10^{-6}$ & This paper \\
Helix nebula & $1.1 \times 10^{4}$ & $4 \times 10^{-7}$ & $(5),(6)$ \\
\hline
\end{tabular}

References. (1) Chesneau et al. (2006); (2) Chesneau et al. (2007); (3) De Marco et al. (1997); (4) Guerrero et al. (2004); (5) Meaburn et al. (2008); (6) Su et al. (2007).

be destroyed by the intense UV radiation field. Chesneau et al. (2006) suggests that an efficient dissipating process is occurring in the disk of CPD $-56^{\circ} 8032$.

The original masses of the disks are unknown. However, disks around post-AGB stars have dust masses similar to that of CPD-56 8032 , for RU Cen and AC Her of $5 \times 10^{-4}$ and $2 \times 10^{-4} M_{\odot}$, respectively (Gielen et al. 2007). These objects have stars that are still too cool to cause ionization, and have avoided ionization-driven dissipation.

Thus, we suggest that the disk of the Helix nebula may be the remnant of a post-AGB dust disk, rather than a debris disk as proposed by Su et al. (2007). Once the star is on the cooling track, the disk becomes largely stable and the additional dissipation time is set by Poynton-Robertson drag, which has a timescale of order $10^{7} \mathrm{yr}$. Thus, the disks may remain detectable for about $10^{8} \mathrm{yr}$, long after any sign of the planetary nebula has disappeared.

Several disks have been discovered around white dwarfs (e.g., Gänsicke et al. 2006). Searches for such disks around the oldest white dwarfs have been unsuccessful (Kilic et al. 2009), but about $15 \%$ of local white dwarfs contain metal-rich material in their photosphere, indicative of accretion from a residual dust reservoir (Sion et al. 2009). Although these white dwarfs may have a variety of origins, they may include the progeny of the M 2-29-type disks.

\section{Conclusions}

We have presented a detailed study of a Galactic bulge planetary nebula, to identify evidence of disk evolution. Our main findings are:

1. M2-29 probably has a distance consistent with membership of the Galactic bulge. It shows absorption features of a cloud with high velocity, which we tentatively identify with the nearby HVC 002.9-06.2-088.

2. The nebula is surrounded by a partial ring, which slowly expands at $12 \mathrm{~km} \mathrm{~s}^{-1}$. This structure may be produced by interaction with the interstellar medium, but based on the expansion, and the alignment with the inner and outer nebula, we associate it with an early mass-loss phase from the central star. A model capable of explaining this structure is one in which mass loss is triggered initially only during periastron of a companion in an elliptical orbit, where the ring was ejected with a preferential velocity in the orbital plane that reflects the orbital motion of the central star.

3. We find evidence of ongoing mass loss from the central source that decreases with time at a slow expansion velocity of $10 \mathrm{~km} \mathrm{~s}^{-1}$, with a current rate of $\sim 10^{-8} M_{\odot} \mathrm{yr}^{-1}$. There is however no evidence of any mass-losing star that could be the source of this material.

4. There is evidence of a very fast wind $\left(\sim 10^{3} \mathrm{~km} \mathrm{~s}^{-1}\right)$ from the central star, based on the $\mathrm{H} \alpha$ line wings. The [O III] also shows wings, but a factor of ten narrower.

5. The unresolved core with diameter $<250$ AU exhibits strong forbidden and permitted emission lines with a range of ionization states, of as low as [OI]. The velocity HWHM of the $4363 \AA$ [O III] line is $18 \mathrm{~km} \mathrm{~s}^{-1}$; [O I] is much narrower. Owing to the very short dynamical timescale and the velocity gradient with excitation, we interpret this as a stable, rotating system. The mass of the core is estimated as $M_{\mathrm{c}} \sim 10^{-4} M_{\odot}$. The dust emission is located within this core.

6. We interpret the ongoing mass loss as a disk wind, driven by the ionization and heating of the gas. The thermal gas velocities are similar to the rotation velocity, making the disk susceptible to evaporation. The fast wind from the star may be accelerating gas shed from the disk, as seen in the [O III] line wings. Both the mass and current disk mass-loss rate are indicative of a decay time similar to the age of the nebula.

7. By comparing the masses of dust disks in planetary nebulae of different ages, we show that the dust disks dramatically reduce their mass as the nebula evolves, reflecting the disk evaporation. The dust mass decrease from $\sim 5 \times 10^{-4} M_{\odot}$ at the start of the PN phase, to $\sim 4 \times 10^{-7} M_{\odot}$ for the helix nebula on the white-dwarf cooling track where the luminosity of the star quickly drops by a factor of 100 , reducing the rate at which the dust disk would be lost.

Acknowledgements. This work was financially supported by MNiSW of Poland through grant No. N20302431/3879. The VLT/UVES observations are from ESO program 075.D-0104, the VISIR data from 077.D-0652 and the archival data came from program 077.D-0652.

\section{References}

Alexander, R. D. 2008, MNRAS, 391, L64

Ben Bekhti, N., Richter, P., Westmeier, T., \& Murphy, M. T. 2008, A\&A, 487, 583

Bond, H. E., Meakes, M. G., Liebert, J. W., \& Renzini, A. 1993, in Planetary nebulae, ed. R. Weinbergerv \& A. Acker (Dordrecht: Kluwer Academic Publishers), IAU Proc. Symp., 155, 499

Borkowski, K. J., Harrington, J. P., \& Tsvetanov, Z. I. 1995, ApJ, 449, L143 Brinkworth, C. S., Gänsicke, B. T., Marsh, T. R., et al. 2009, ApJ, 696, 1402 Casassus, S., Roche, P. F., Aitken, D. K., \& Smith, C. H. 2001, MNRAS, 327, 744

Chesneau, O., Collioud, A., de Marco, O., et al. 2006, A\&A, 455, 1009

Chesneau, O., Lykou, F., Balick, B., et al. 2007, A\&A, 473, L29

Cohen, M., Barlow, M. J., Liu, X.-W., \& Jones, A. F. 2002, MNRAS, 332, 879

Costero, R., Tapia, M., Méndez, R. H., et al. 1986, Rev. Mex. Astron. Astrofis., 13,149

Dame, T. M., Hartmann, D., \& Thaddeus, P. 2001, ApJ, 547, 792

Dekker, H., D’Odorico, S., Kaufer, A., Delabre, B., \& Kotzlowski, H. 2000, Proc. SPIE, 4008, 534

de Marco, O. 2009, PASP, 121, 316

de Marco, O., Barlow, M. J., \& Storey, P. J. 1997, MNRAS, 292, 86

de Marco, O., Barlow, M. J., \& Cohen, M. 2002, ApJ, 574, L83

de Ruyter, S., van Winckel, H., Maas, T., Lloyd Evans, T., et al. 2006, A\&A, 448,641

Dominik, C., Dullemond, C. P., Cami, J., \& van Winckel, H. 2003, A\&A, 397, 595

Durand, S., Acker, A., \& Zijlstra, A. 1998, A\&AS, 132, 13

Exter, K. M., Barlow, M. J., \& Walton, N. A. 2004, MNRAS, 349, 1291

Gänsicke, B. T., Marsh, T. R., Southworth, J., \& Rebassa-Mansergas, A. 2006, Science, 314, 1908

Galazutdinov, G. 2005, JKAS, 38, 215

Garcia-Rojas, J., Esteban, C., Peimbert, A., et al. 2005, Rev. Mex. Astron. Astrofis., 24, 243

Gesicki, K., \& Zijlstra, A. A. 2000, A\&A, 358, 1058

Gesicki, K., \& Zijlstra, A. A. 2003, MNRAS, 338, 347 
K. Gesicki et al.: Disk evaporation in a planetary nebula

Gesicki, K., Zijlstra, A. A., Acker, A., et al. 2006, A\&A, 451, 925

Gielen, C., van Winckel, H., Waters, L. B. F. M., Min, M., \& Dominik, C. 2007, A\&A, 475, 629

Gill, D. 1900, Annals of the Cape Observatory, 5, 1

Guerrero, M. A., Chu, Y.-H., \& Miranda, L. F. 2004, AJ, 128, 1694

Hajduk, M., Zijlstra, A. A., \& Gesicki, K. 2008, A\&A, 490, L7

Howard, J. W., Henry, R. B. C., \& McCartney, S. 1997, MNRAS, 284, 465

Kilic, M., Kowalski, P. M., Reach, W. T., \& von Hippel, T. 2009, ApJ, 696, 2094

Krist, J., \& Hook, R. 2004, The Tiny Tim User's Guide V6.3 (Baltimore: STScI)

Lagadec, E., Zijlstra, A. A., Matsuura, M., et al. 2008, MNRAS, 383, 399

Lamers, H. J. G. L. M., Zickgraf, F.-J., de Winter, D., Houziaux, L., \& Zorec, J. 1998, A\&A, 340, 117

Manchado, A., Guerrero, M. A., Stanghellini, L., \& Serra-Ricart, M. 1996, The IAC morphological catalog of northern Galactic planetary nebulae (La Laguna, Spain: Instituto de Astrofisica de Canarias (IAC)), Foreword by S. R. Pottasch, ISBN: 8492180609

Meaburn, J., López, J. A., \& Richer, M. G. 2008, MNRAS, 384, 497

Megier, A., Strobel, A., Bondar, A., et al. 2005, ApJ 634, 451

Mihalas, D., 1971, ApJ, 170, 541

Minniti, D., \& Zoccali, M. 2008, in Galactic Bulges, ed. M. Bureau, et al., IAU Symp., 245, 323

Miszalski, B., Acker, A., Moffat, A. F. J., Parker, Q. A., \& Udalski, A. 2009, A\&A, 496, 813
Nordhaus, J., \& Blackman, E. G. 2006, MNRAS, 370, 2004

Pasquini, L., Avila, G., Blecha, A., et al. 2002, The Messenger, 110, 1

Phillips, J. P., \& Ramos-Larios, G. 2008, MNRAS, 383, 1029

Putman, M. E., de Heij, V., Staveley-Smith, L., et al. 2002, AJ, 123, 873

Ramos-Larios, G., Phillips, J. P., Richer, M. G., \& Kemp, S. N. 2008, MNRAS, 387, 415

Rodriguez, M., Corradi, R. L. M., \& Mampaso, A. 2001, A\&A, 377, 1042

Sandquist, E. L., Taam, R. E., Chen, X., et al. 1998, ApJ, 500, 909

Sion, E. M., Holberg, J. B., Oswalt, T. D., McCook, G. P., \& Wasatonic, R. 2009, AJ, 138, 1681

Selvelli, P., Danziger, J., \& Bonifacio, P. 2007, A\&A, 464, 715

Soker, N., \& Rappaport, S. 2001, ApJ, 557, 256

Su, K. Y. L., Chu, Y.-H., Rieke, G. H., et al. 2007, ApJ, 657, L41

Torres-Peimbert, S., Dufour, R. J., Peimbert, M., \& Peña, M. 1997, in Planetary nebulae, Proceedings of the 180th Symposium of the International Astronomical Union (IAU), ed. H. J. Habing, \& H. J. G. L. M. Lamers (Kluwer Academic Publishers)

van Hoof, P. A. M., Hajduk, M., Zijlstra, A. A., et al. 2007, A\&A, 471, L9

Van Winckel, H., Schwarz, H. E., Duerbeck, H. W., \& Fuhrmann, B. 1994, A\&A, 285,241

Villaver, E., García-Segura, G., \& Manchado, A. 2002, ApJ, 571, 880

Wareing, C. J., Zijlstra, A. A., \& O'Brien, T. J. 2007, MNRAS, 382, 1233

Zickgraf, F.-J. 2003, A\&A, 408, 257 